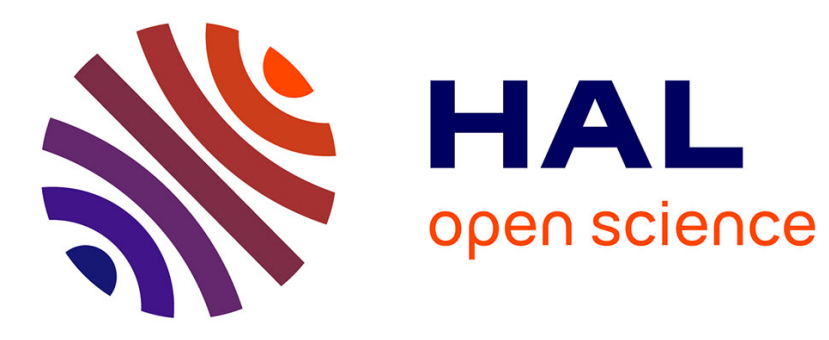

\title{
Object-oriented processing of CRM precipitation forecasts by stochastic filtering
}

Philippe Arbogast, Olivier Pannekoucke, Laure Raynaud, Renaud Lalanne, Etienne Mémin

\section{- To cite this version:}

Philippe Arbogast, Olivier Pannekoucke, Laure Raynaud, Renaud Lalanne, Etienne Mémin. Objectoriented processing of CRM precipitation forecasts by stochastic filtering. Quarterly Journal of the Royal Meteorological Society, 2016, 142 (700), pp.2827-2838. 10.1002/qj.2871 . hal-01378366

\section{HAL Id: hal-01378366 \\ https://hal.inria.fr/hal-01378366}

Submitted on 21 Oct 2016

HAL is a multi-disciplinary open access archive for the deposit and dissemination of scientific research documents, whether they are published or not. The documents may come from teaching and research institutions in France or abroad, or from public or private research centers.
L'archive ouverte pluridisciplinaire HAL, est destinée au dépôt et à la diffusion de documents scientifiques de niveau recherche, publiés ou non, émanant des établissements d'enseignement et de recherche français ou étrangers, des laboratoires publics ou privés. 


\title{
Object-oriented processing of CRM precipitation forecasts by stochastic filtering
}

\author{
Philippe Arbogast ${ }^{1}$, Olivier Pannekoucke ${ }^{1}$, Laure Raynaud ${ }^{1}$, Renaud Lalanne ${ }^{1}$ and Etienne \\ Mémin $^{2}$ \\ ${ }^{1}$ CNRM/GAME, Météo-France/CNRS URA 1357, Toulouse, France \\ ${ }^{2}$ INRIA, Vista Project, Center of Rennes \\ ${ }^{*}$ Correspondence to: Philippe Arbogast, Météo-France CNRM/GMAP, 42 Av. G. Coriolis, 31057 Toulouse Cedex, \\ France. E-mail : philippe.arbogast@meteo.fr
}

In order to cope with small-scale unpredictable details of mesoscale structures in cloud-resolving models, it is suggested in this paper to process the model outputs following a fuzzy object-oriented approach to extract and track precipitating features (associated with a higher predictability than the direct model outputs). The present approach uses the particle filter method to recognize patterns based on predefined texture or spatial variability of the model output. This provides an ensemble of precipitating objects, which are then propagated in time using a stochastic advection-diffusion process. This method is applied to both deterministic and ensemble forecasts provided by the AROME-France convective-scale model. Specific case studies support the ability of the approach to handle precipitation of different types. Copyright (c) 2015 Royal Meteorological Society

Key Words: Particle filter, precipitation forecast, fuzzy object, ensemble prediction

$\underline{\text { Received .. }}$

Citation: .... 


\section{Introduction}

The lack of dense observing networks for dynamical variables as well as microphysical ones leads to a relative poor predictability in current Non-Hydrostatic models at the $\mathrm{km}$ scale resolution. Although these models succeed in simulating a wide variety of convective situations, they eventually fail to represent mesoscale features at the appropriate location or appropriate time, and with the correct size and structure. Thus, advanced spatial methods have been proposed in the recent past for verifying mesoscale forecasts.

The first straightforward but qualitative method that allows to cope with such errors is to check the model against observations "by eye". On the other hand, objective approaches include neighborhood methods, which compare forecasts and observations within space-time windows of varying sizes (Ebert, 2008). One example is upscaling (Zepeda-Arce et al., 2000; Yates et al., 2006), where forecasts and observations are averaged to coarser grid resolutions, thus filtering out the noisy unskillful higher-resolution scales. The fractions skill score method of Roberts and Lean (2008) compares the fractional occurrence of rain exceeding a threshold within a neighborhood. In Atger (2001), the notion of multiple thresholds related to the distance of the forecast event to the observations is introduced. Scale separation is another filtering method used to distinguish between predictability at different scales, based on the application of singlebandpass spatial filters such as wavelet transforms (Briggs and Levine (1997).

Another interesting approach is to consider forecasts and observations as objects, as in Ebert and McBride (2000). This technique imitates the intuitive human approach by identifying rain features, usually focusing on relatively large scales so that the fine-scale errors have lesser importance. Most of the methods detect features by applying a threshold to the fields (Davis et al, 2006). Cluster analysis, as proposed by, e.g, Marzban and Sandgathe (2006, 2008), Michaes et al. (2007), can also be used to identify relevant patterns. In that framework, it then becomes possible to decompose forecast errors into position, size, shape and amplitude errors (e.g., SAL method in Wernli et al., 2008), and decision models can be introduced to decide what is a satisfactory forecast (Damrath, 2004 ; Nachamkin, 2004 ; Baldwin and Kain, 2006). Finally, some authors are also able to exhibit position error fields using the optical flow technique (Marzban and Sandgathe, 2010, Keil and Craig, 2007 ; 2009).

The purpose of the present paper is to introduce a novel method for detection and tracking of precipitating objects in mesoscale forecasts, based on the following main features : (i) the method runs at a coarser resolution than the model itself, (ii) the object is defined by the probability density function of the precipitation field inside the object, summarizing the rich forecast signal at full horizontal resolution, (iii) the object is a closed smooth curve which is tracked using a displacement field provided by an optical flow technique, (iv) uncertainties regarding the shape and location of the object are introduced by the use of a stochastic filter (Avenel et al., 2009). It is then expected that weak (respectively large) uncertainty of the object edge location in case of simple shape (respectively complicated) precipitation patterns will be obtained.

The paper is organized as follows. The definition of an object and the detection-tracking algorithm are presented in section 2. The detection algorithm is first applied in section 3 to deterministic forecasts from the convective-scale AROME-France model in order to demontrate its ability to successfully recognize precipitation patterns of different types. The tracking algorithm is then illustrated on a case of severe thunderstorms. Finally, the application of the object-oriented approach to ensemble forecasts provided by the convective-scale AROME ensemble prediction system (EPS) is examined. Section 5 provides conclusions and future work. 

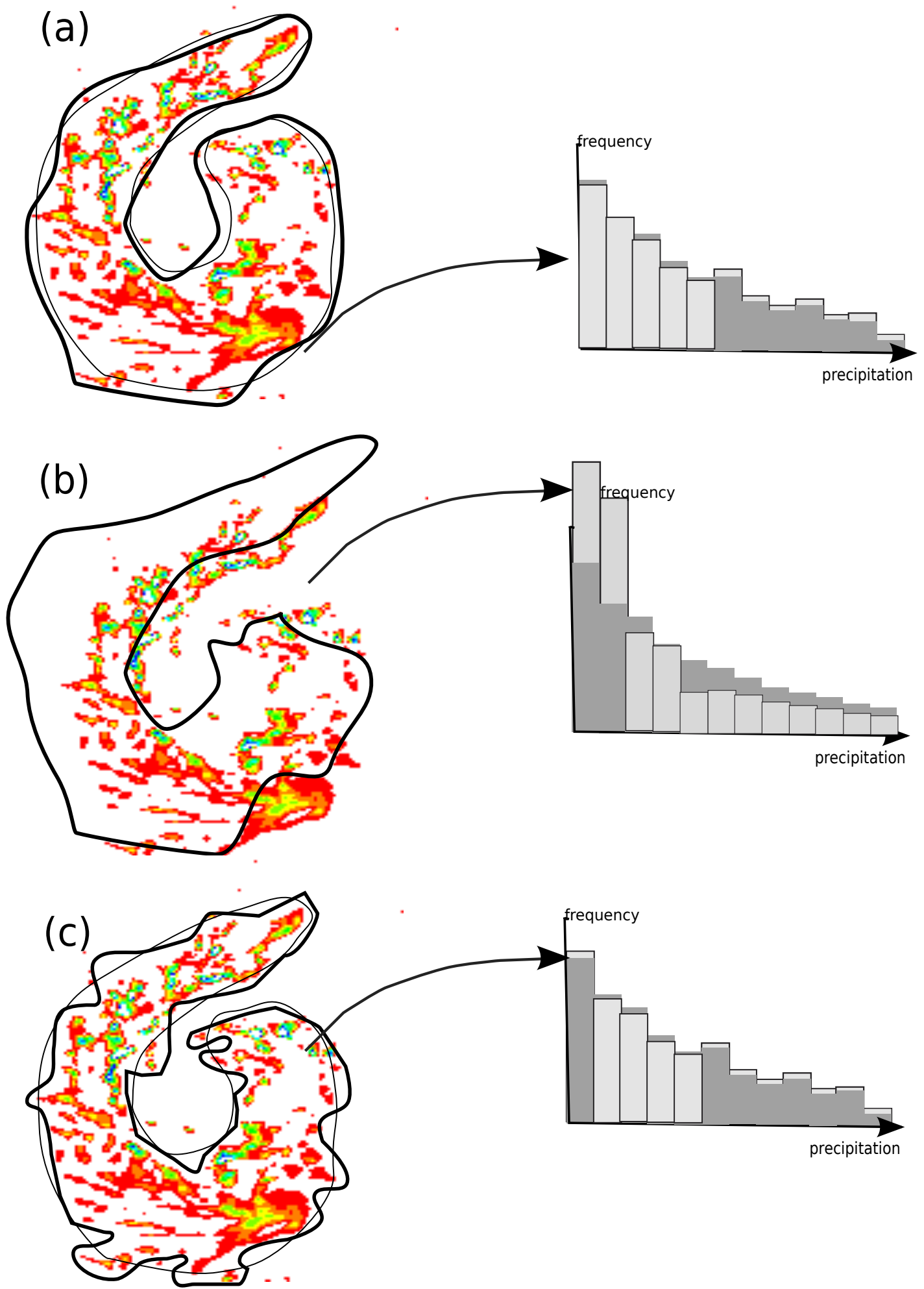

FIGURE 1. (a) : Two slightly different objects with comparable frequency histograms. (b) : object whose shape is comparable with (a) but with significant space shift. The corresponding histogram is now different from the original one with an excess of weak precipitation. (c) here two objects with close histograms but with different level of smoothness.

2. Definition, detection and dynamics of a fuzzy precipitating object

\subsection{Definition of a fuzzy precipitating object}


nature, namely with either the same precipitation amount or, more generally, with more or less the same variability over the whole area (see Figure 1). It will be assumed hereafter that the precipitation histogram provides an estimation of the spatial variability. We also assume to be able to follow the object along time. The method is meant for objects with moderate time evolution of their shape, including splitting and merging.

In the remainder of the paper, a precipitating object at time $t$ is defined as a simple closed curve $\mathcal{C}_{t}$ that encompasses mesoscale precipitation patterns. The main criterion used to discriminate the nature of the precipitating object is the precipitation histogram $h$ : cold-air convective areas, mesoscale convective systems (MCSs) or even frontal structures are associated with different histograms. An object that encompasses a single MCS would be associated with an histogram with a peak at moderate to heavy precipitation rates whereas a large-scale object with eventually several MCSs would be associated with a flatter histogram. Figure (a) shows an example of a precipitation pattern whose shape presents a larger scale with respect to the individual precipitating features. It is possible to draw subjectively, as suggested by the Figure, some contours with close precipitation distributions and therefore with the same likelihood, whereas a space shift as shown in panel (b) leads to a different precipitation distribution inside an unlikely contour. In the sense of the precipitation distribution the contour presented in panel (c) is again likely. However, it is noisy and therefore it should not be allowed by the detection algorithm.

To handle a $1 \mathrm{D}$ contour $\mathcal{C}_{t}$, it turns out to be more convenient to consider it as an isoline of a 2D surface $(\phi(x, y, t))$, called a level set function (Osher and Sethian, 1988; Sethian, 1996). The contour is then defined as the zero level set :

$$
\mathcal{C}_{t}=\left\{\mathbf{x}_{t}(s): \phi\left(\mathbf{x}_{t}(s), t\right)=0\right\}
$$

where $s$ stands for a parameter of the curve and $\mathbf{x}_{t}=(x, y)$. The zero level set is sufficient as a definition of the contour of interest. As in Avenel et al. (2009), one now consider the level set function as the signed distance function to the contour of interest, defined as

$$
\phi(\mathbf{x}, t)=\left\{\begin{array}{l}
-d\left(\mathbf{x}, \mathcal{C}_{t}\right) \text { if } \mathbf{x} \text { is inside } \mathcal{C}_{t} \\
d\left(\mathbf{x}, \mathcal{C}_{t}\right) \text { if } \mathbf{x} \text { is outside } \mathcal{C}_{t}
\end{array}\right.
$$

where $d\left(\mathbf{x}, \mathcal{C}_{t}\right)$ is the distance between the location denoted by $\mathbf{x}$ and the closest point belonging to the contour. Then $d\left(\mathbf{x}, \mathcal{C}_{t}\right)=\min _{\mathbf{x}_{t}(s) \in \mathcal{C}_{t}}\left\|\mathbf{x}-\mathbf{x}_{t}(s)\right\|$ where $\|$.$\| stands for$ the $L_{2}$-norm. Figure shows a case of structure splitting, a configuration where the level-set approach is really powerful. Splitting thunderstorms are examples of such situations.

While it is easy to perceive coherent structures by eye, a full precise mathematical description is still a challenge. The basic idea of our method is to overcome this mathematical difficulty by introducing the concept of fuzzy object : the object is considered as resulting from a stochastic process with a certain degree of randomness, and it is described by an ensemble of simple curves $\mathcal{C}_{t}^{k}$, $k \in[1, N]$ where $N$ is the size of the ensemble. This probabilistic approach then allows us to account for some uncertainties related to assumptions in the detection and tracking steps. More details regarding the generation of the ensemble will be given in subsequent sections.

\subsection{Nonlinear filtering of the contour set}

The aim of the method is to perform detection and tracking of objects characterized by some features, here the precipitation histogram within the objects. For that purpose, predefined reference histograms corresponding to some particular types of precipitation are initially specified and Bayesian estimation is used to search for the most likely objects given the reference histograms.

Bayesian filters constitute well-known procedures to estimate the posterior probability density function (pdf 
(a)

(b)

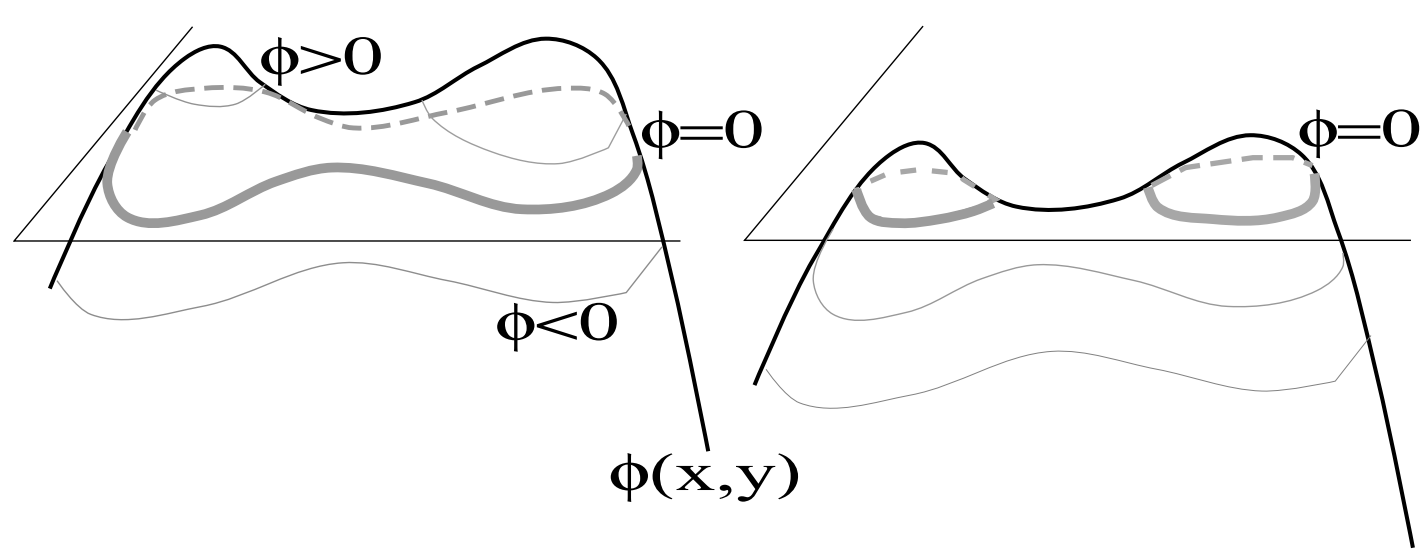

FigURE 2. Evolution of the $\phi$ function during a splitting event. The heavy grey line stands for the object contour. (a) : One object is present. (b) two objects appear.

hereafter) $p\left(\mathcal{C}_{t} \mid \mathbf{y}_{1: t}\right)$ (called the filtering distribution) of a state variable $\mathcal{C}_{t}$ at any measurement instant $t$, given the discrete measurements series $\mathbf{y}_{1: t}=\left(\mathbf{y}_{1}, \ldots, \mathbf{y}_{t}\right)$ until instant $t$, and an initial distribution $p\left(\mathcal{C}_{0}\right)$. Here, $\mathcal{C}_{t}$ is a closed curve featured by the zero level of a level set function and the measurements are provided by the reference histograms.

The inference of the posterior pdf may be obtained in two successive stages : a prediction step and a correction step. The prediction uses the transition distribution $p\left(\mathcal{C}_{t} \mid \mathcal{C}_{r<t}\right)$ to achieve a first approximation of the next state. Then, the correction step updates the posterior pdf using the Bayes theorem :

$$
p\left(\mathcal{C}_{t} \mid \mathbf{y}_{1: t}\right) \propto p\left(\mathbf{y}_{t} \mid \mathcal{C}_{t}\right) p\left(\mathcal{C}_{t} \mid \mathbf{y}_{1: t-1}\right)
$$

It appears that the likelihood $p\left(\mathbf{y}_{t} \mid \mathcal{C}_{t}\right)$ of a new measurement $\mathbf{y}_{t}$ obtained at instant $t$ is central to this problem.

Particle filters (PF) are Monte Carlo techniques specifically designed for sequential Bayesian estimation when systems are nonlinear and non-Gaussian (Gordon et al. 1993, Doucet et al. 2000, Del Moral, 2004, Van Leeuwen, 2009, Baehr and Pannekoucke, 2011). In that framework, the posterior distribution $p\left(\mathcal{C}_{t} \mid \mathbf{y}_{1: t}\right)$ is approximated by a finite weighted sum of $N$ Diracs centred on hypothesized locations in the state space, which are called particles (here a particle is a simple curve) :

$$
p\left(\mathcal{C}_{t} \mid \mathbf{y}_{1: t}\right)=\sum_{k=1}^{N} w_{t}^{k} \delta\left(\mathcal{C}_{t}-\mathcal{C}_{t}^{k}\right)
$$

where $\mathcal{C}_{t}^{k}$ is the $k^{t h}$ particle, $\delta$ the Dirac function and

$$
w_{t}^{k}=\frac{p\left(\mathbf{y}_{t} \mid \mathcal{C}_{t}^{k}\right)}{\sum_{l=1}^{N} p\left(\mathbf{y}_{t} \mid \mathcal{C}_{t}^{l}\right)}
$$

is a weight applied to the $k^{t h}$ particle, that depends on the observations likelihood. A large (resp. small) weight will be assigned to a good (resp. bad) particle. Although particles of the ensemble are in interaction, the large size of the ensemble $(\mathcal{O}(100))$ leads to convergence of the empirical pdf toward a limit pdf (Del Moral, 2004).

\subsection{Metrics used in the likelihood in the case of image processing}

A key aspect in particle filtering is the definition of the observation likelihood $p\left(\mathbf{y}_{t} \mid \mathcal{C}_{q}^{t}\right)$, which directly determines the weights of each particle. In this work, the likelihood is computed based on similarity between a precipitation distribution of reference and the precipitation distributions in the regions delineated by the current curve sample. 
Since we consider here precipitation model outputs as images we use the Bhattacharyya (1943) coefficient to measure the overlap beween an object histogram $h_{k}$ to the prescribed reference histogram $h_{r e f}$ instantiated at time 0 . Assume $h_{\text {ref }}$ is defined with $p$ classes $: h_{\text {ref }}=$ $\left(h_{r e f}^{1}, \ldots, h_{r e f}^{p}\right)$ normalized so that $\sum_{i} h_{r e f}^{i}=1$, and $h_{k}=$ $\left(h_{k}^{1}, \ldots, h_{k}^{p}\right)$ denotes the histogram associated with $\mathcal{C}_{t}^{k}$, the Bhattacharyya coefficient is given by :

$$
b_{k}=\sum_{i} \sqrt{h_{r e f}^{i} h_{k}^{i}} .
$$

A distance can be defined as :

$$
d_{k}=\left(1-b_{k}\right)^{1 / 2}
$$

The weights are then computed following equation (3), with the observation likelihood defined as :

$$
p\left(\mathbf{y}_{t} \mid \mathcal{C}_{t}^{k}\right) \propto \exp \left(-\lambda d_{k}\right)
$$

where $\lambda$ is a positive parameter. In the application presented below $\lambda=1$. The results are weakly sensitive to the choice of $\lambda$ in pratice.

\subsection{Detection algorithm}

The object detection algorithm is implemented through a histogram-based particle filter and a level-set representation, as described in the previous sections. The filter is initialized with an ensemble of random level set functions $\phi^{k}(\mathbf{x}, t)$, which are obtained by convolving a realization of a 2D normal distribution $\mathcal{N}(0,1)$ with an auto-correlation function $\rho(x, y)=\exp \left\{\frac{-\left(x^{2}+y^{2}\right)}{2 L^{2}}\right\}$, where $L$ is a characteristic length-scale that depends on the size of the object to be detected. Since precipitation patterns are defined to be the regions where $\phi^{k}>0$, histograms of precipitation are calculated on each positive connected component of the field. Each histogram is then weighted according to its distance to the prescribed reference histogram, as explained in section .

The object of interest is, at the end, the expected contour of the random object $\mathcal{C}_{t}$. It is exhibited by the zero level set of the weighted ensemble mean, defined as $\bar{\phi}(\mathbf{x}, t)=\sum_{k}, w_{t}^{k} \phi^{k}(\mathbf{x}, t)$. The uncertainty associated with this structure is given by the weighted local variance of the level set functions around the mean level set : $v\left(\mathbf{x}_{s}\right)=$ $\sum_{k} w_{t}^{k} \phi^{k}\left(\mathbf{x}_{s}, t\right)^{2}$ where $\mathbf{x}_{s}$ is a given point of the mean curve. The term related to the square of the mean vanishes since the mean is zero by definition along the mean contour. This variance defines the width of the uncertainty band : the lower the variance the narrower the uncertainty band. This band helps visualize the regions where the curve's sample exhibits some variability. This variability arises from some approximations in the detection process, including for instance the number of particles used to run the filter, the definition of the likelihood function and the coarser working grid resolution. It also depends on the data : it is higher in regions where the likelihood is poorly defined, such as regions far away from the reference histograms.

Precipitating areas delineated by the mean contour are found to be in relatively good agreement with the prescribed histograms, but they generally lack accuracy regarding the shape of the precipitating objects. In order to determine a contour that segments the precipitation field in a meaningful way, the Chan-Vese (hereafter CV) segmentation algorithm (Chan and Vese, 2001) is then applied to the mean levelset function. The purpose of the CV segmentation is to partition a given image into two regions, one representing the objects to be detected and the other representing the background. It is based on an energy minimization problem, which can be formulated using the level set formalism. The basic idea is to evolve the level set function representing the contours according to some evolution equation until it reaches a steady state that provides a useful segmentation of the image. The algorithm usually converges in a few iterations. We refer the reader to Chan and Vese (2001) 
for a detailed presentation of the CV algorithm. Figure presents the contour of a detected object before and after the CV segmentation. As expected, the segmentation process reconstructs an object whose shape is in better agreement with the precipitation field.

In a final step, the uncertainty associated with the detection process, featured by the variance $v$, is accounted for by generating an ensemble of contours around the segmented mean contour. This is achieved by adding random perturbations of variance $v$ to the segmented mean contour.

Compared to the thresholding methods commonly used for the detection of precipitating objects (e.g. Davis et al., 2006 ; Ebert 2008), no fixed precipitation threshold is used to define the objects. It is replaced by a set of reference histograms that allows for the identification of objects of different types. The specified histograms are then expected to be an important issue in this detection algorithm. It may also be noted that the present approach provides two additional outcomes : first, the fuzzy nature of the algorithm helps to figure out the spatial uncertainty associated with the detection process; secondly, a user can directly get from the object identification a classification of the precipitation patterns at play.

\subsection{Tracking algorithm}

The second part of our PF-based method is the tracking of the detected objects. For that purpose, the time evolution of a contour $\mathcal{C}_{t}$ is chosen so that it resembles the phase propagation of a wave front. In addition, the uncertainty in the modelling of this dynamics is represented with two independent Brownian motions directed along the contour's tangent and normal respectively. Appendix A shows that the level set function $\phi$ is then the solution of an advectiondiffusion equation with a stochastic forcing :

$$
\partial_{t} \phi+(\mathbf{c} \cdot \mathbf{n})|\nabla \phi|=\frac{\sigma^{2}}{2} \Delta \phi
$$

where $\mathbf{c}$ is a displacement vector field (see Figure for an example) between two time steps, which is retrieved from an optical flow computation, and $\mathbf{n}$ is the normal to the contour. The right-hand side term accounts for the uncertainty on the curve dynamics and $\sigma$ is the amplitude of the applied perturbations. Equation (4) is then used in the prediction step of the particle filter to propagate forward in time each particle (i.e. each contour $\mathcal{C}_{t}^{k}$ of the ensemble).

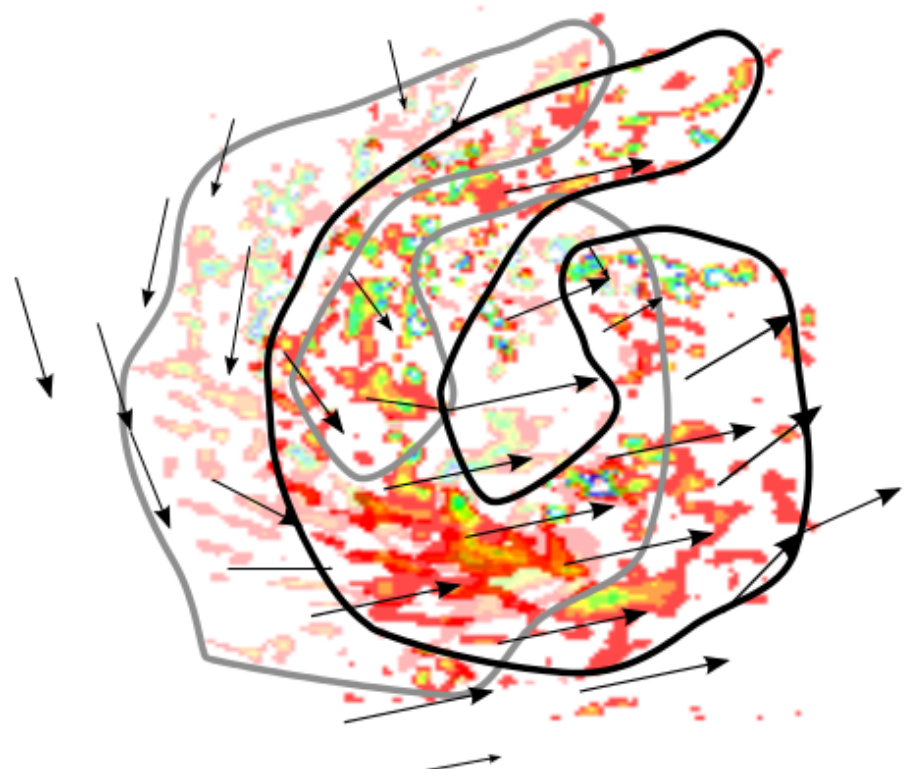

FigURE 4. Displacement field associated with the evolution of the light shaded patterns towards the heavy shaded ones.

\subsection{Summary of an iteration of the filter}

The initialization of the procedure corresponds to the specification of the reference histograms associated with the predefined precipitation categories. The detection/tracking algorithm is then applied in parallel for each reference histogram, as summarized by Figure; the list below refers to the figure :

Step 0 : specification of reference precipitation histograms

Step 1 : detection of objects based on particle filtering and Chan-Vese segmentation algorithm. Outcomes are one sample of contours for each reference histogram; 


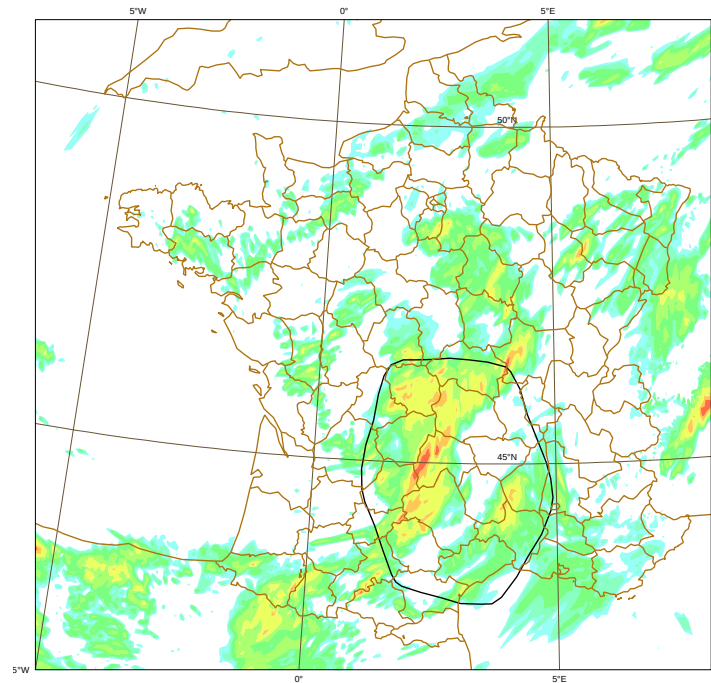

(a) initial contour

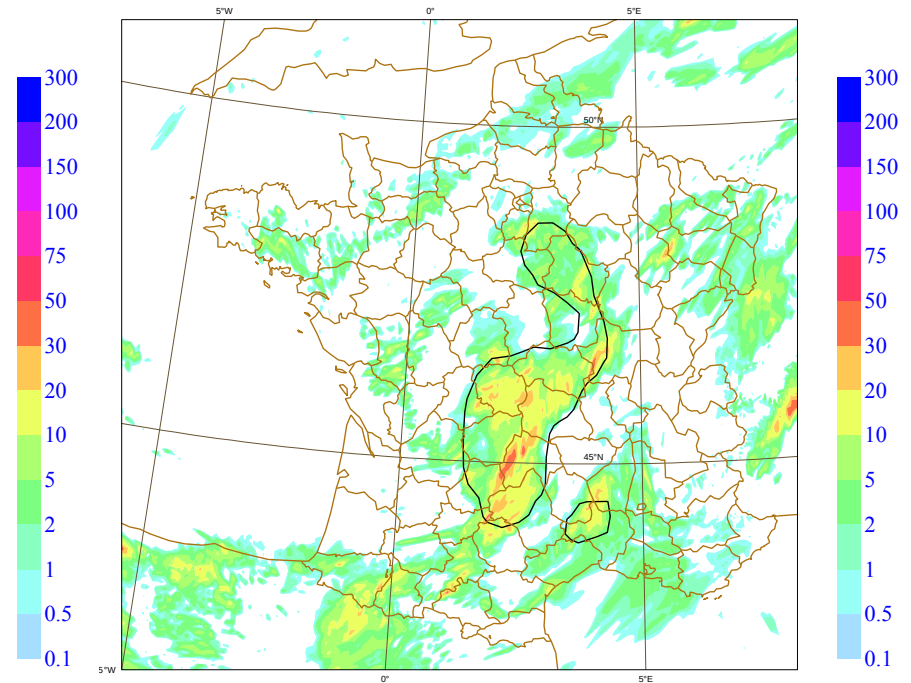

(b) contour after segmentation

FIGURE 3. 3-hourly accumulated precipitation (mm) forecasted by the AROME-France model from 15 to 18 UTC on 26 April 2015 . Contour of the object detected (a) before and (b) after the Chan-Vese segmentation algorithm.

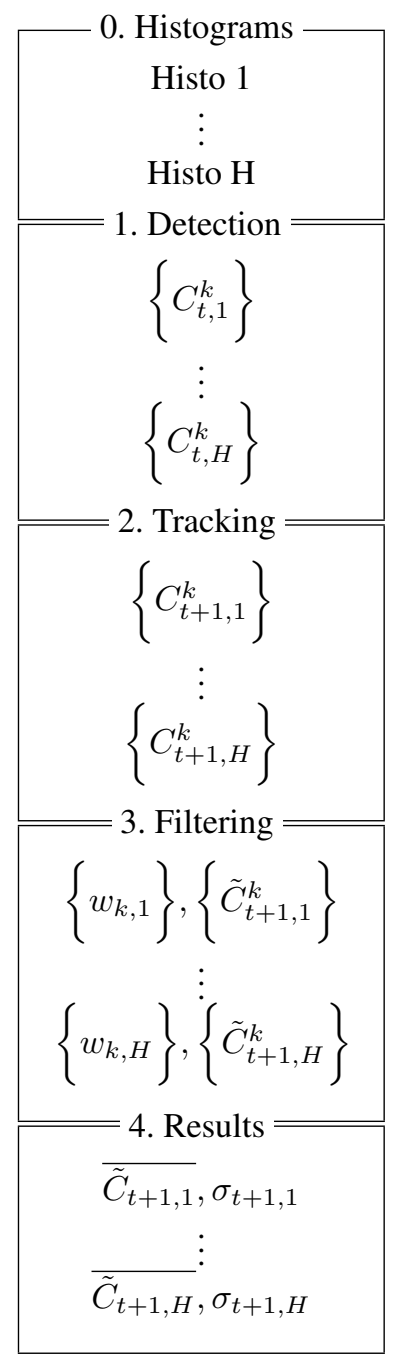

FIGURE 5. Schematic of the detection/tracking algorithm for one timestep.
Step 2 : evolution of each sample of contours using the stochastic advection-diffusion model;

Step 3 : weights computation and ensemble resampling;

Step 4 : determination of the objects, i.e. expectation, best contour and uncertainty band using the resampled ensemble ;

Steps 1 to 4 are repeated until the end of the tracking period.

It may be mentioned that, since the detection/tracking algorithm is applied in parallel for each prescribed histogram, then 1) we don't need to choose a category in advance and 2) similar objects can be detected in more than one category. This second aspect could be avoided by improving the reference histograms or by using a more discriminative likelihood function. It can also be interpreted as an indication of uncertainty regarding the type of precipitation at play.

\subsection{Some tuning issues}

The level of randomness introduced by $\sigma$ in contour's dynamics (equation (4)) is supposed to represent the non-conservativity of the precipitation time-sequence and should also be related to the spatial scales allowed by 
the smoothness constraint which is introduced within the variational optical flow method. Two different approaches will be considered hereafter to address the tuning of $\sigma$.

In the first one, $\sigma$ corresponds to the spread of the ensemble of particles along the contour's tangent and normal. If its distribution after resampling is significantly modified with respect to the prescribed values it is considered that the distribution of $\sigma$ must be adjusted. This approach has been adopted to tune the method for the case study presented in the following section.

Appendix B presents a second approach based on the following assumption : the noise associated with the level set displacement and the curve noises are collinear and have the same variances.

\section{Results}

\subsection{Experimental set-up}

The detection and tracking algorithms are applied in this section to precipitation forecasts from the AROME-France model. The AROME model (Seity at al., 2011) is a spectral, non-hydrostatic convective-scale model which has been running operationally at Météo-France since December 2008 over French territory. In this paper, experiments are performed with a model configuration that uses either a $2.5 \mathrm{~km}$ or a $1.3-\mathrm{km}$ horizontal resolution. The analysis step is performed with a $3 \mathrm{D}$-Var scheme.

The approach is also applied to an ensemble prediction system based on the AROME-France model with a $2.5-\mathrm{km}$ horizontal resolution, which is currently being developed at Météo-France for operational forecasting. This ensemble is made of 12 members designed to take into account uncertainties in the initial and boundary conditions, the model and surface fields (Bouttier et al., 2012; Bouttier et al., 2014 ; Raynaud and Bouttier 2014).

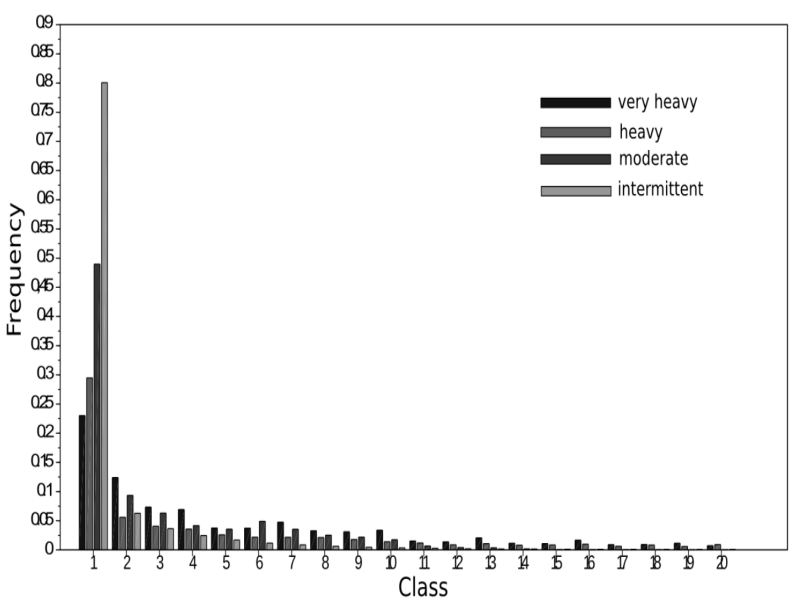

FIGURE 6. 20 first classes of the reference histograms of precipitation prescribed for the detection/tracking algorithm. They correspond to very heavy precipitation (black), heavy precipitation (medium gray), moderate precipitation (dark gray) and intermittent precipitation (light gray). A class corresponds to $0.23 \mathrm{~mm} / \mathrm{h}$.

\subsection{Object detection}

Three case studies have been selected to test the object detection method and examine its ability to discriminate precipitation of various types. The detection is performed using a number of particles fixed as 100, and four histograms have been prescribed (Figure ), corresponding to very heavy precipitation, heavy precipitation, moderate precipitation and intermittent precipitation. These histograms have been computed on a set of past events and use 100 classes. An ensemble of 50 contours is then generated from the mean level-set function in order to take into account the fuzzy nature of the detected objects.

\subsubsection{Severe thunderstorms}

Severe thunderstorms developed on 26 April 2015 in a relatively large area along a South West/North East axis over France, leading locally to heavy precipitation and hail. The initialization of the object using a 3-hour precipitation accumulation from a $18 \mathrm{~h}$ forecast of the AROMEFrance model has been successful with the histogram corresponding to heavy precipitation. Figure displays the initial state of the object, which is actually an ensemble of 


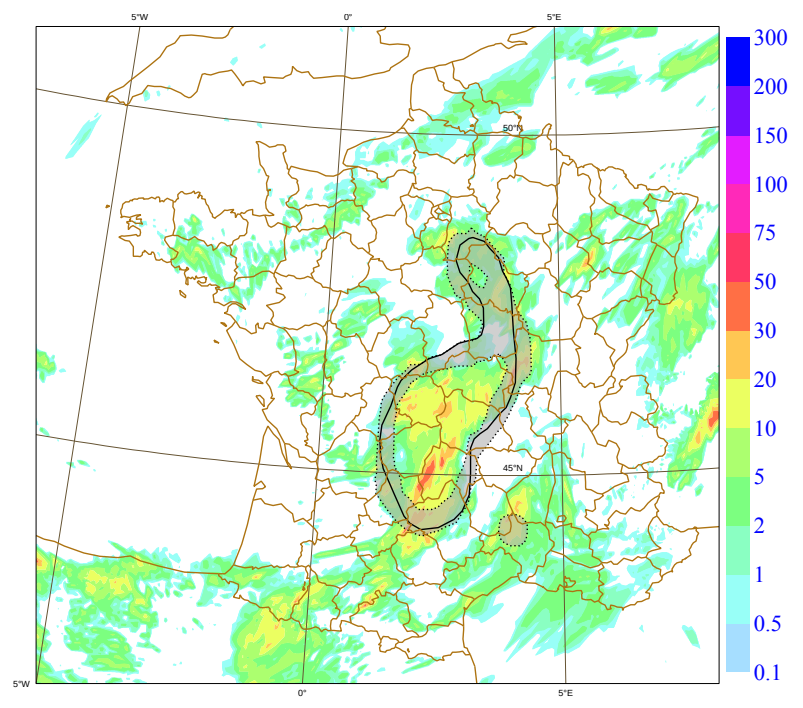

FIGURE 7. 3-hourly accumulated precipitation (mm) from 15 to 18 UTC on 26 April 2015, from a 18h forecast of the AROME-France model, along with the best initial detected object (black solid) and the area where the ensemble members are lying (the shaded strip).

$N=50$ contours. The ensemble is summarized by the best contour (regarding the reference histogram) and the area (the shaded strip) where the ensemble members are lying. The horizontal extension of that shaded strip measures the uncertainty behind the object determination. The detected object well reflects the identification that could have been done "by eye". Moreover, the area of the object with smaller precipitation is characterized by a larger uncertainty, thus reflecting that the data does perfectly match the reference histogram in this region.

\subsubsection{Heavy precipitating event}

The ability of the approach to detect a small area of very high amount of rainfall, resulting locally in severe flooding, is examined on the case of the heavy precipitating event that occured over Southeastern France on 17 September 2014. Figure shows that the object of interest is well detected in the 6-hour precipitation accumulation from a $18 \mathrm{~h}$ forecast using the histogram corresponding to very heavy precipitation.

\subsubsection{Intermittent precipitation}

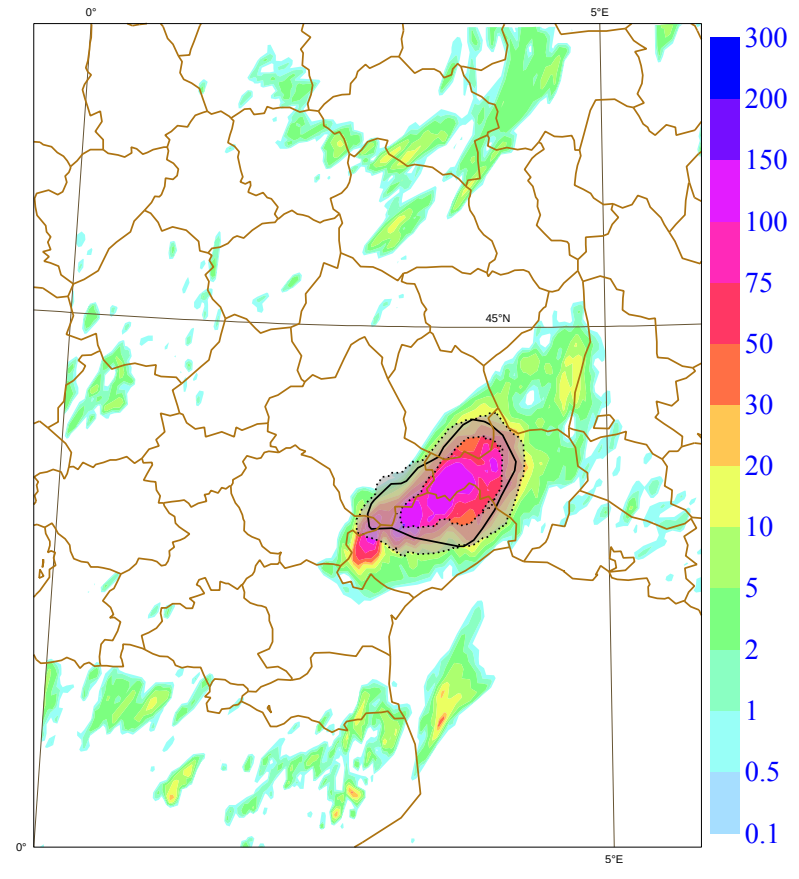

FIGURE 8. 6-hourly accumulated precipitation (mm) from 12 to 18 UTC on 17 September 2014, from a 18h forecast of the AROME-France model, along with the best initial detected object (black solid) and the area where the ensemble members are lying (the shaded strip).

The performance of the detection algorithm regarding intermittent precipitation is investigated here, considering a 1-hour precipitation accumulation from a $14 \mathrm{~h}$ forecast valid on 21 February 2015, where an active stormy sky occured after the passage of a cold front. It is characterized by an ensemble of small convective structures with varying size and intensity. Such spatially discontinuous features are particularly interesting since traditional thresholding methods could have difficulties to treat them as one feature. Figure indicates that the proposed detection method is able to accurately extract the whole precipitating areas. It can also be noted that the uncertainty associated with this object is larger than in the other two examples.

\subsection{Tracking : a case study}

In a second step, the tracking algorithm is tested on a case of severe thunderstorms that hit North-Eastern France on 10 June 2014. The initialization of the tracking corresponds to the detection of precipitating objects in a 1hour precipitation accumulation forecast from the AROME model valid at 20 UTC. Three distinct convective areas are 


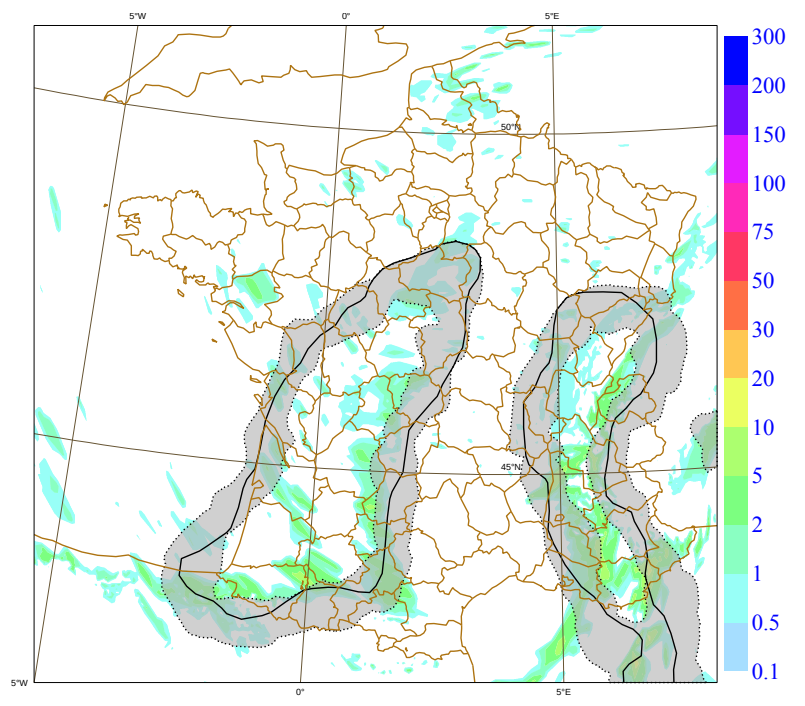

FIGURE 9. 1-hourly accumulated precipitation (mm) from 13 to 14 UTC on 21 February 2015, from a 14h forecast of the AROME-France model, along with the best initial detected object (black solid) and the area where the ensemble members are lying (the shaded strip).

identified (Figure (a)).

Optical flows along the sequence of forecasts are also required. They are computed following the algorithm for large displacement optical flow estimation from Steinbrücker et al. (2009).

As explained in section $2(\mathrm{~g})$, a critical parameter of the tracking is the level of noise associated with the two Brownian motions introduced within the stochastic advection-diffusion model. Weak noise could prevent the filter from representing large local uncertainties but the pdf of the error could be documented with a relatively small ensemble. Strong noise could enable the stochastic filter to represent strong uncertainty. In that latter case the main drawback is the size of the ensemble which must be large enough. In the present experiment, the level of noise is adjusted after each iteration of the particle filter, according to the variance of the resampled ensemble.

Figures (b)-(f) present the temporal evolution of the initial convective cells over a 5h-period. The tracking algorithm accurately follows the objects throughout the forecast period. It is interesting to note that the method is able to deal with splitting and merging, for instance, at timestep 1 the algorithm correctly merges the two northern cells, with a large uncertainty in the area without precipitation. The decay of the southern cell is also well captured by the algorithm : at timestep 2 this object is only present in a small number of members, while no more members forecast it from timestep 3. The method thus appears robust to track moderate-moving features with nonnegligeable distortion.

The results from the tracking can be summarized by calculating strike probability for tracked objects (e.g. Dance (2010)), that is, the probability that a given gridpoint will be affected by a precipitating object within a forecast time period. Denoting $T$ the length of the forecast period, the strike probability is defined by

$$
P^{T}(\mathbf{x})=\frac{1}{T} \frac{1}{N} \sum_{l=1}^{T} \sum_{k=1}^{N} P_{l}^{k}(\mathbf{x})
$$

$$
\text { where } P_{l}^{k}(\mathbf{x})=\left\{\begin{array}{l}
1 \text { if } \mathbf{x} \text { is inside } \mathcal{C}_{l}^{k} \\
0 \text { if } \mathbf{x} \text { is outside } \mathcal{C}_{l}^{k}
\end{array}\right.
$$

Figure presents the strike probability corresponding to the 5h-tracking with an ensemble of 50 objects at each timestep. This map shows a strong chance that thunderstorms affect the eastern part of Belgium.

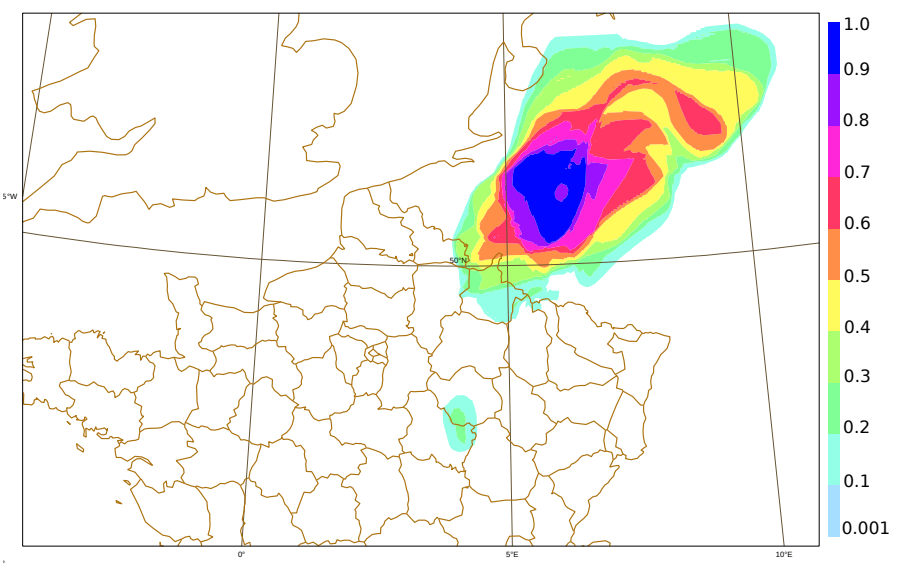

FIGURE 11. Probability that a given gridpoint will be affected by a precipitating object between 20 UTC on 10 June 2014 and 01 UTC on 11 June 2014, calculated from the stochastic tracking of convective cells identified in the AROME-France forecast valid at 20 UTC on 10 June 2014. 


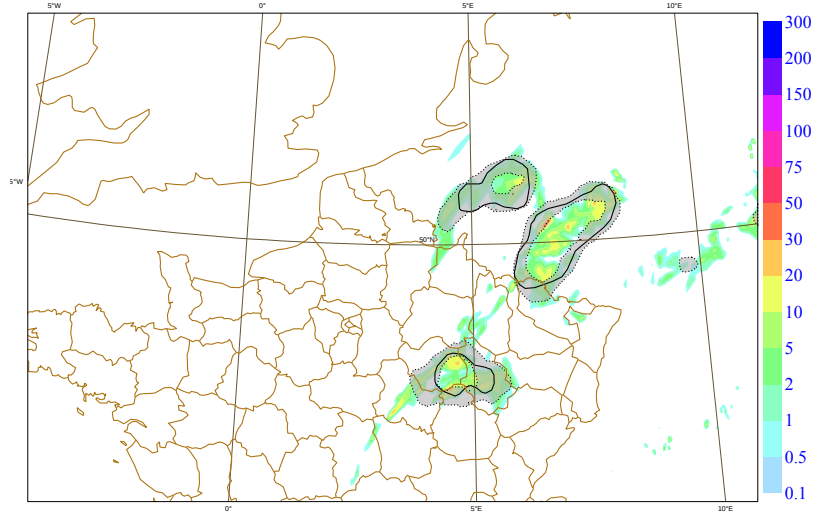

(a) initialization

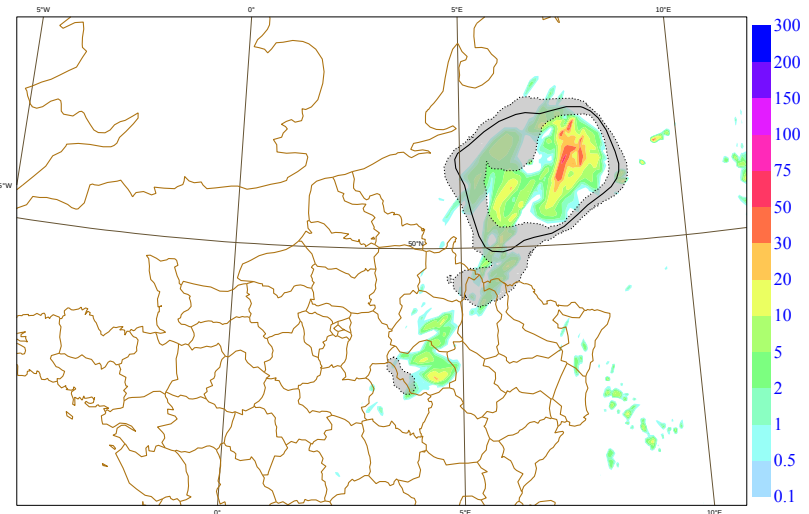

(c) $\mathrm{T}+2 \mathrm{~h}$

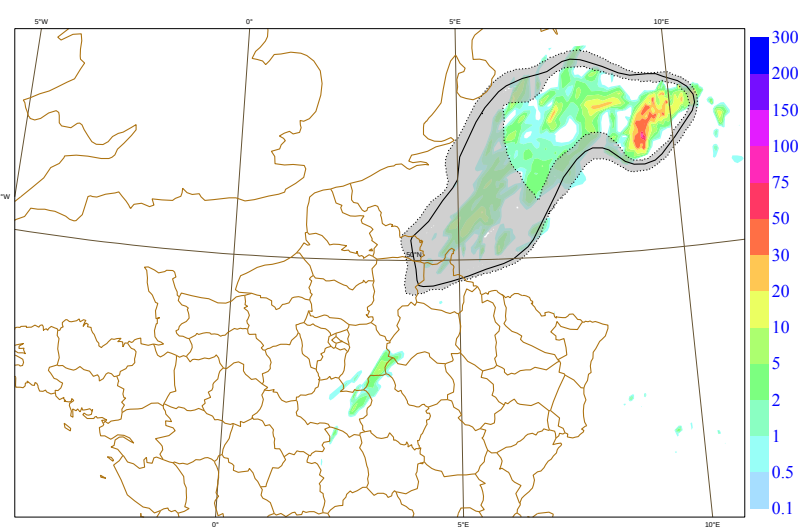

(e) $\mathrm{T}+4 \mathrm{~h}$

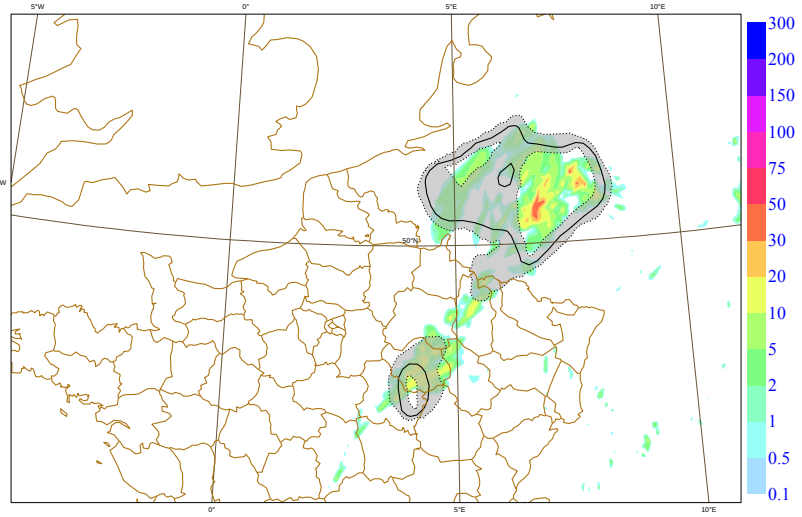

(b) $\mathrm{T}+1 \mathrm{~h}$

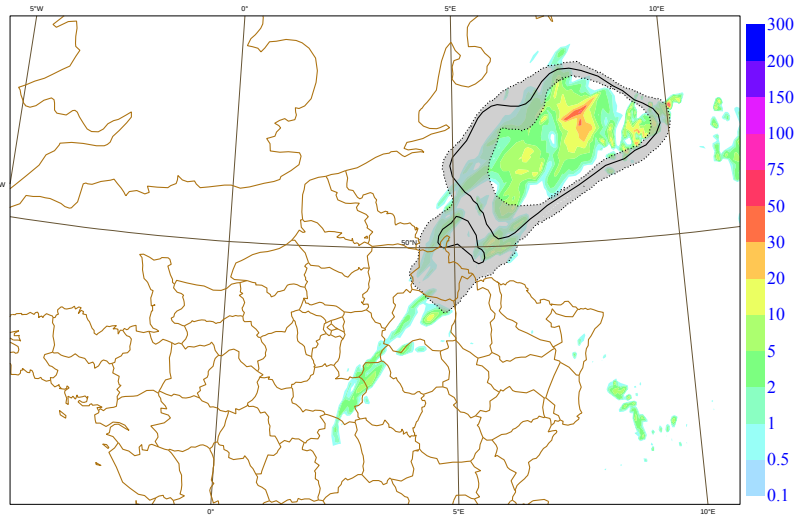

(d) $\mathrm{T}+3 \mathrm{~h}$

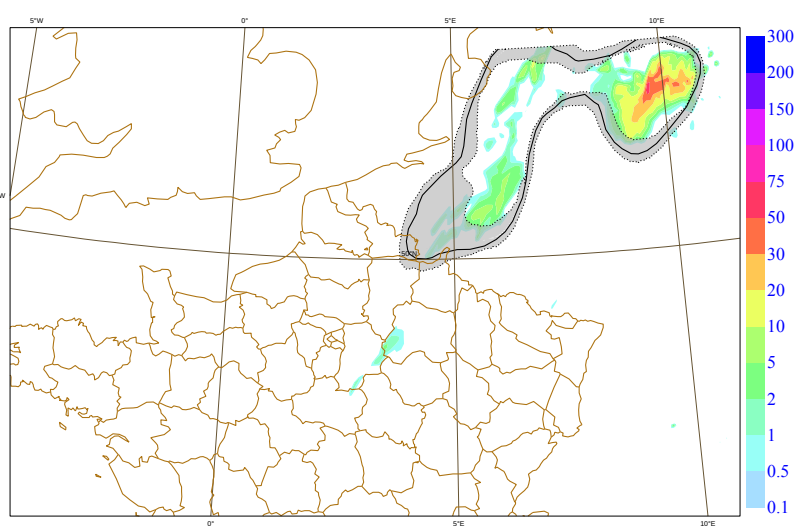

(f) $\mathrm{T}+5 \mathrm{~h}$

FIGURE 10. (a) 1-hourly accumulated precipitation (mm) forecasted by the AROME-France model valid at 20 UTC on 10 June 2014 , along with the best initial detected object (black solid) and the area where the ensemble members are lying (the shaded strip). (b)-(f) Result of the tracking over a 5h period, summarized by the mean contour (black solid) and the area covered by the resampled ensemble (the shaded strip).

\subsection{Application to ensemble forecasts}

An ensemble prediction system provides a variety of scenarios which characterize the uncertainty of the forecast. However, the relatively small sizes of current operational ensemble forecasts at convective scale somewhat limit their utility. In this context, post-processing techniques such as the neighborhood method and the time-lagging approach (Ben Bouallègue et al., 2013 ; Raynaud et al., 2014), which are based on the introduction of a spatial and/or temporal tolerance in model outputs, have been proposed to increase the value of ensemble forecasts. Object-oriented techniques are another way to focus on precipitating features rather than gridpoint-based values. The proposed detection-tracking algorithm which has 


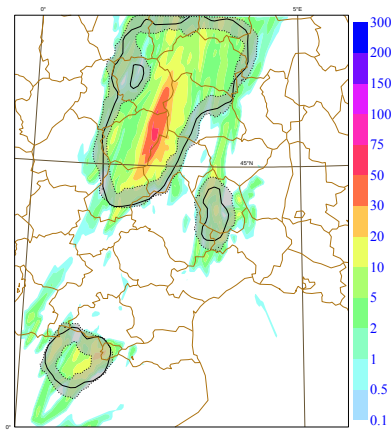

(a) $\mathrm{mb} 1$

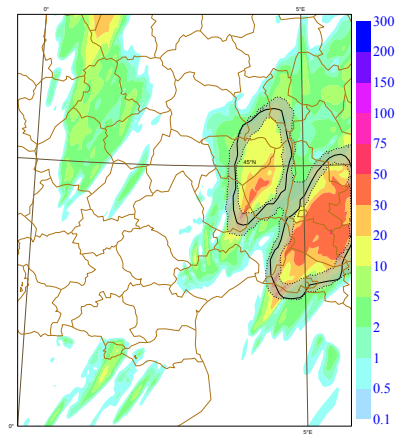

(e) $\mathrm{mb} 5$

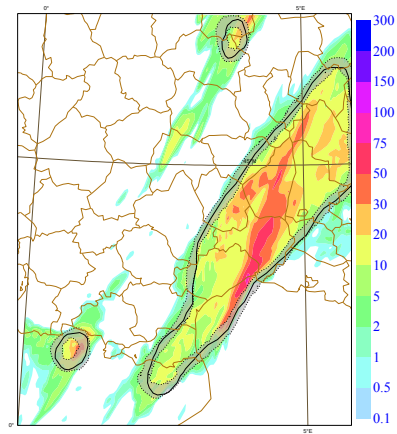

(i) $\mathrm{mb} 9$

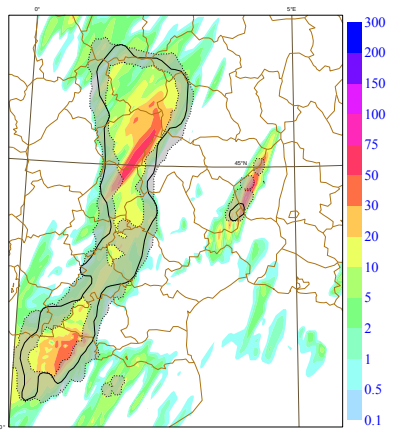

(b) $\mathrm{mb} 2$

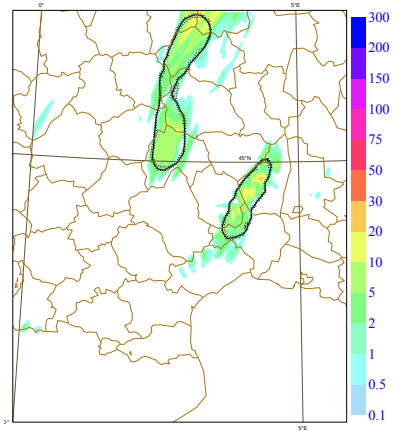

(f) $\mathrm{mb} 6$

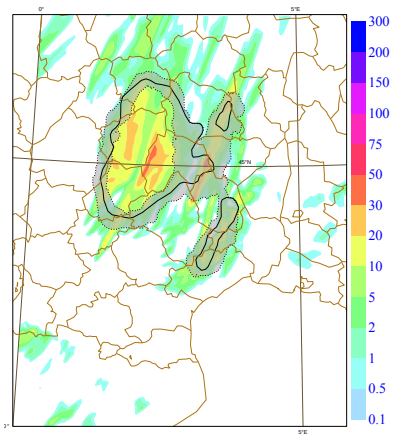

(j) $\mathrm{mb} 10$

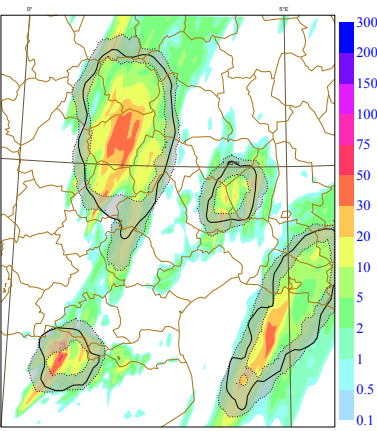

(c) $\mathrm{mb} 3$

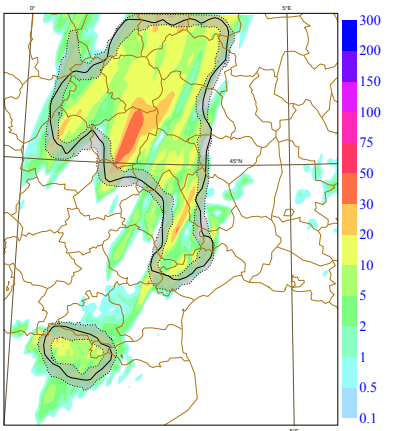

(g) $\mathrm{mb} 7$

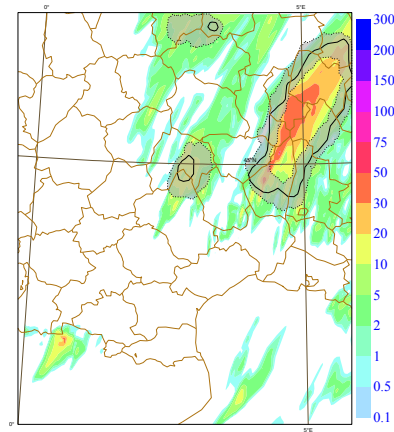

(k) mb 11

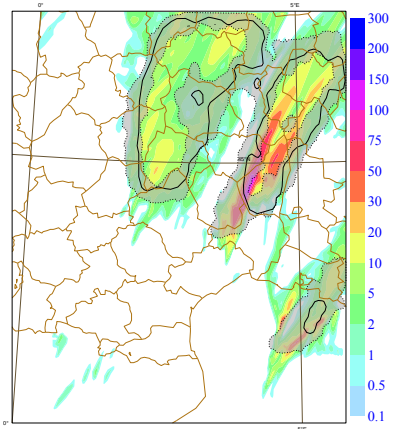

(d) $\mathrm{mb} 4$

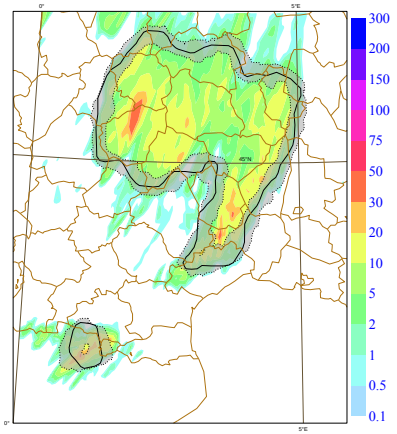

(h) $\mathrm{mb} 8$

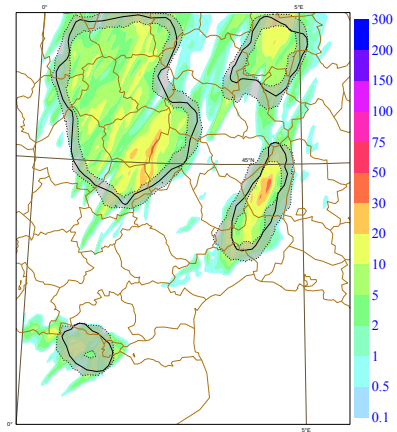

(1) $\mathrm{mb} 12$

FIGURE 13. $24 \mathrm{~h}$ forecasts of 6-hourly accumulated precipitation (mm) between 15 and 21 UTC on 12 October 2014 . Each panel presents the forecast of individual members of the AROME ensemble prediction system.

initially been developed for deterministic forecasts can also be applied to each member of an ensemble system. This provides a 'super-ensemble' of precipitating objects, which combines the sampling provided by the EPS and the sampling associated with the fuzzy implementation of the object processing.

The detection part of the method has been applied to an ensemble of 12 perturbed AROME forecasts for a case of heavy precipitation that occured on 12 october 2014 over Southeastern France. Of particular interest is the line of heavy rainfall locally exceeding $100 \mathrm{~mm}$ in 6 hours (see observations in Figure ). Figure shows the twelve 24hour forecasts of 6-hour precipitation accumulation, along with the results from the objects identification. The objects extracted by the algorithm fall in the categories heavy or very heavy precipitation depending on the member, and look broadly similar to the features a user could detect "by eye". In that sense, the object identification scheme could provide a useful help to forecasters when analyzing precipitating features forecasted by an EPS. Looking either at the precipitation fields or at the detected objects, one can observe relatively large differences regarding 


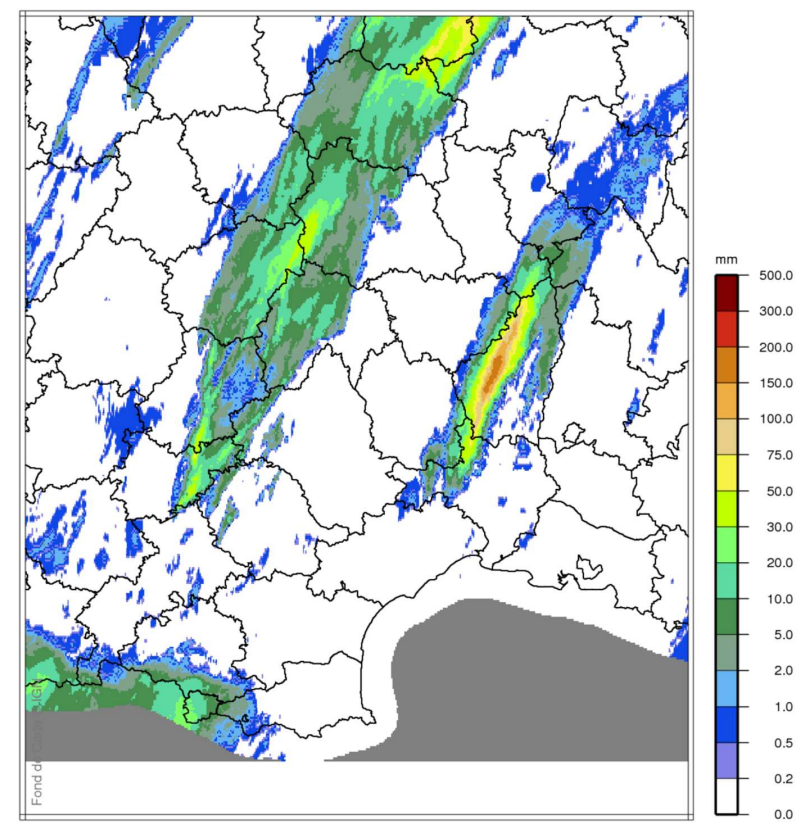

FIGURE 12. 6-hourly accumulated precipitation ( $\mathrm{mm}$ ) between 15 and 21 UTC on 12 October 2014, observed by radar data and rain gauges.

the localization, size, shape and intensity of precipitating patterns forecasted by each member of the ensemble. A consequence of this variability is the necessity to apply specific thresholds for each member when using traditional thresholding methods to detect objects. Hence, developing threshold-free approaches may be particularly relevant for a convective-scale EPS.

The information provided by the detection step can be summarized by calculating object probabilities over the $N$ available contours. At each gridpoint, the object probability is calculated as the probability of being inside a precipitating object :

$$
\begin{gathered}
P(\mathbf{x})=\frac{1}{N} \sum_{k=1}^{N} P^{k}(\mathbf{x}), \\
\text { where } P^{k}(\mathbf{x})=\left\{\begin{array}{l}
1 \text { if } \mathbf{x} \text { is inside } \mathcal{C}_{t}^{k} \\
0 \text { if } \mathbf{x} \text { is outside } \mathcal{C}_{t}^{k}
\end{array}\right.
\end{gathered}
$$

On the one hand, these probabilities can be calculated considering all detected objects. On the other hand, since the detection step provides a classification of the precipitating patterns, the probabilities can be calculated

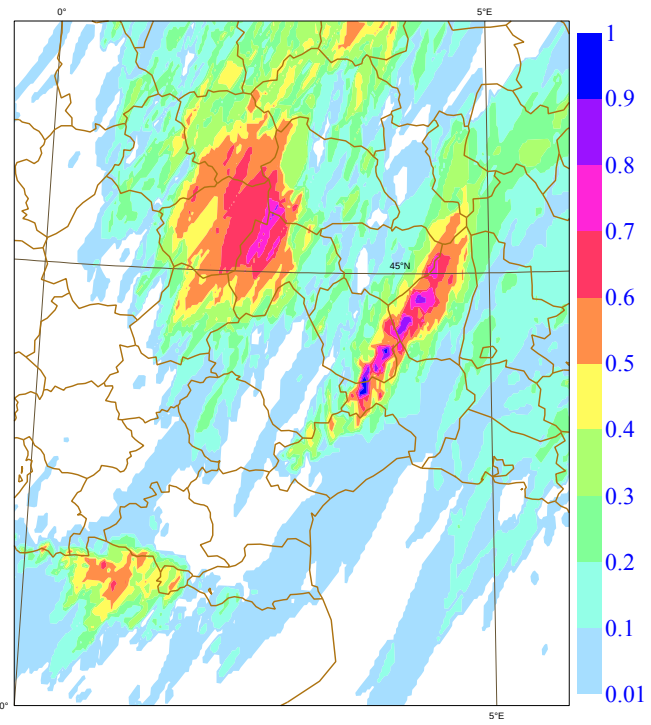

(a) Gridpoint probabilities $r r 6 \mathrm{~h}>5 \mathrm{~mm}$

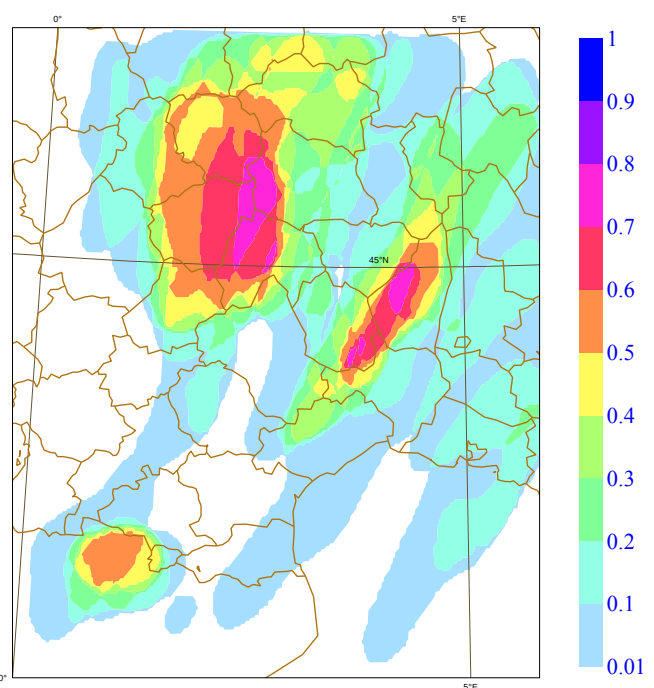

(b) Dynamical object probabilities

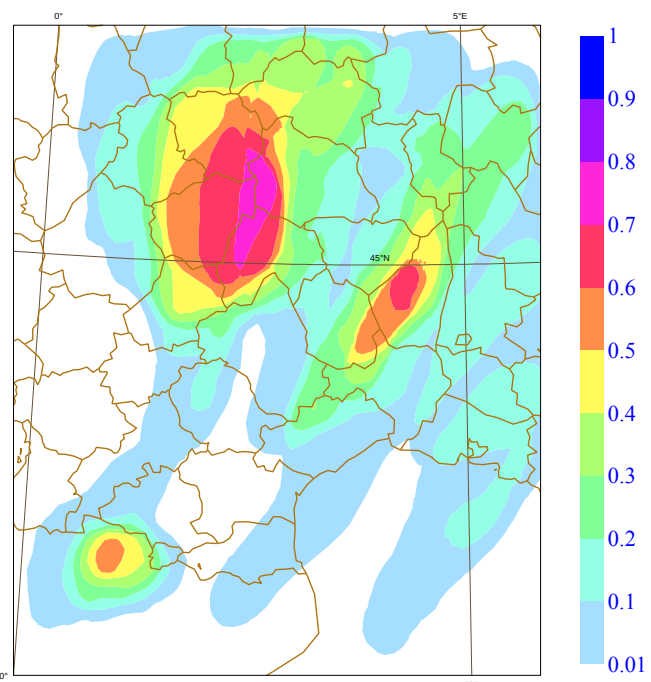

(c) Dynamical/stochastic object probabilities

FigURE 14. Probabilities calculated from the 24h AROME ensemble forecasts of 6-hourly accumulated precipitation, valid on 12 October 2014 at 21 UTC. (a) Gridpoint probabilities for 6-hour precipitation exceeding $5 \mathrm{~mm}$, (b)-(c) object probabilities calculated using the best object and the ensemble of objects for each member respectively. 
for a particular type of object. Compared to traditional gridpoint probabilities, object probabilities at a particular gridpoint do not rely on the forecast at that gridpoint but on the nature of the forecast within a spatial neighborhood defined by the identified precipitating patterns. Hence, the object probability can be seen as a particular case of neighborhood probability where the neighborhood is objectively and adaptively defined.

Object probabilities have been first calculated using deterministic objects, chosen as the best contours detected in each member of the ensemble ( $N=12$ in that case). As indicated by Figure (b), these probabilities provide an accurate signal for the localization of heavy precipitation, with higher values in the regions of observed intense rainfall (Figure ). The comparison with gridpoint probabilities of precipitation exceeding $5 \mathrm{~mm}$ in 6 hours (Figure (a)) supports the idea that the object-oriented processing of precipitation has a similar smoothing effect as spatial neighborhood methods and allows one to more clearly visualize the localization of heavy rainfall.

Object probabilities have then been calculated based on fuzzy objects, using the ensemble of 50 extracted contours for each member $(N=12 \times 50=600$ in that case, Figure (c)). These probabilities correspond to a smoothed version of the raw object probabilities and less high probabilities are forecasted. In addition, accounting for uncertainties in the object detection is shown to improve the forecast to some extent. For instance, regarding the eastern object Figure (b) indicates two distinct areas with probabilities higher than $70 \%$, while observations show that only one is actually concerned by heavy rainfall. In contrast, only the correct region is highlighted in the probabilities calculated from the fuzzy objects.

Object probabilities have been calculated based on the fuzzy objects detected in the $6 \mathrm{~h}$ accumulated precipitation field of each member. In order to represent the uncertainty in the time evolution of the precipitation over this 6h-period, these probabilities could also be derived from the ensembles of tracked objects, by applying the tracking algorithm to each member over the 6h-period, similarly to what has been done on a deterministic forecast in the previous section.

\section{Conclusions}

An object-based method for the detection and tracking of precipitation features has been developed and applied to deterministic and ensemble forecasts provided by a cloudresolving model. It is based on the particle filter approach with a level-set representation of closed curves, including precipitation histograms to characterize the patterns and stochastic laws for their temporal evolution.

Compared to common object-oriented detection approaches (e.g. Davis et al., 2006 ; Ebert 2008), an advantage of the proposed detection algorithm is that no fixed precipitation threshold is required to define the objects. This is particularly interesting when applying the algorithm to a convective-scale ensemble prediction system that often provides very different forecasts. Instead, the detection step relies on a set of prescribed reference histograms that allows for the identification of objects of different types, in terms of both intensity and spatial variability.

The primary motivation for applying a particle filter approach in this work is its ability to estimate precipitation patterns in conjunction with associated uncertainties. In addition, it is a first step towards adaptive tracking, since the amplitude of the noise applied in the stochastic advection model can be tuned according to the variance of the particle set. In the end, the location of the edge of each object, along with the associated uncertainty band, are possible useful outcomes of the method.

The detection algorithm is shown to work well on a variety of precipitation fields. In particular, its performance regarding the detection of highly intermittent precipitation, such as those encountered in stormy skies, is encouraging.

Illustration of the tracking model on a case of severe thunderstorms also provides promising results, with a correct handling of merging/splitting and growth/decay of 
convective cells. Future research will further investigate the potential of the method for probabilistic precipitation nowcasting. The possibility to avoid the computation of the optical flow and to learn the dynamics from the past trajectories (Avenel et al., 2014) will also be examined.

Although the object-oriented detection/tracking method has been initially developed for deterministic forecasts, the present study gives first insight into the way the approach could be applied to ensemble forecasts. In particular, a relevant result may be provided by object probabilities which, in contrast to traditional gridpoint probabilities, focus on scales where the model can be expected to be accurate (Bowler et al., 2006). From that point of view, object probabilities can be seen as a particular case of spatialized probabilities computed over neighborhoods whose size and shape directly depend on the local precipitating features.

It is likely that the present approach could be applied in various other applications such as the tracking of volcanic ash, fog, mid-latitude windstorms, tropical cyclones in numerical models as well as in satellite or radar observations.

It is worth emphasizing that the success of the method relies on the quality of the prescribed reference histograms to a large extent. Future work will examine the impact of defining additional categories in order to improve the discrimination of precipitation patterns. Moreover, the possibility to use flow-dependent instead of climatological histograms, through an iterative search of precipitation patterns, will be explored.

Finally, the probabilistic evaluation of this object-based processing of mesoscale precipitation fields will be the subject of a future study.

\section{Acknowledgments}

The authors acknowledge the support of the French Agence Nationale de la Recherche (ANR), under grant GeoFLUIDS (ANR- 09-SYSC-005-01) "Analyse et simulation d'écoulements fluides à partir de séquences d'images : application à l'étude d'écoulements géophysiques".

\section{Appendix A : deterministic and stochastic model for contour time evolution}

The time evolution of a contour $\mathcal{C}_{t}$ can be understood as the phase propagation of a wave front along characteristic curves $\mathbf{x}_{t}(s)$, of parameter $t$ and fixed $s$. By this way, $\phi\left(\mathbf{x}_{t}(s), t\right)$ appears as a Riemann invariant and thus the conservation of $\phi$ along the characteristic leads to the equation $d \phi=\partial_{t} \phi d t+\nabla \phi \cdot d \mathbf{x}=0$. Hence, the time propagation of the phase is governed by the equation $\frac{d \phi}{d t}=$ $\partial_{t} \phi+\nabla \phi \cdot \frac{d \mathbf{x}}{d t}=0$. Writing $\mathbf{c}=\frac{d \mathbf{x}}{d t}$ the velocity along the characteristics, and $\nabla \phi=|\nabla \phi| \mathbf{n}$ the gradient, where $\mathbf{n}=\nabla \phi /|\nabla \phi|$ is the normal to the levelcontour, the time propagation reduces to the Hamilton-Jacobi equation

$$
\partial_{t} \phi+(\mathbf{c} \cdot \mathbf{n})|\nabla \phi|=0
$$

where $\mathbf{c}$ is a velocity vector estimated using the data through an optical flow method. Since any tangential displacement has no effect on the position of the contour, only the velocity component along the local normal to the level contour plays a role in Eq.(7). Depending on the convexity, the characteristic curves can intersect leading to an overlapping of the contour, associated to an occurence of singularities. Since the resulting curve is not differential, weak solutions are considered. A weak solution is then chosen as the envelope of the curve. This corresponds to a weak entropy solution $\phi_{e}$, obtained as the vanishing viscosity solution $\lim _{\varepsilon \rightarrow 0} \phi_{\varepsilon}$ of the modified dynamics (Osher and Sethian, 1988 ; Sethian, 1996)

$$
\partial_{t} \phi+(\mathbf{c} \cdot \mathbf{n}-\varepsilon \kappa)|\nabla \phi|=0,
$$

in the limit, as the viscosity $\varepsilon$ decreases to zero, and where $\kappa=\nabla \cdot \mathbf{n}=\frac{1}{|\nabla \phi|}\left(\Delta \phi-\frac{\nabla \phi^{T} \phi^{\prime \prime} \nabla \phi}{|\nabla \phi|^{2}}\right)$ is the curvature of the level set isolines, where $\phi^{\prime \prime}$ is the Hessian matrix of $\phi$ 
$\left(\phi_{i j}^{\prime \prime}=\partial_{i j}^{2} \phi\right)$. In numerical application, $\varepsilon$ is set to a small enough value in order to lead to a good approximation of the entropy solution while providing a smooth solution.

Within this approach, the complex dynamics of a simple contour is replaced by the dynamics of a surface, governed by the modified Hamilton-Jacobi equation (MHJE) Eq.(8). This kind of deterministic dynamics has been employed for tracking of curves and motion under a filtering through variational formulation (Papadakis and Mémin, 2008). A stochastic version has been proposed by Avenel et al. (2009). In this framework, the idea is to construct a stochastic dynamics for $\phi$ corresponding to the dynamics described by the MHJE.

To this end, the characteristic curves $\mathbf{x}_{t}(s)$ are replaced by a stochastic Itô diffusion (Oksendal, 2003 ; Pannekoucke and Vezard, 2010)

$$
d \mathbf{X}_{t}=\mathbf{c} d t+\sigma_{1} \mathbf{n} d B_{t}^{1}+\sigma_{2} \mathbf{n}^{\perp} d B_{t}^{2}
$$

where $\mathbf{n}^{\perp}$ stands for the tangent unit vector obtained after a $\pi / 2$ rotation of $\mathbf{n} ; B_{t}^{1}$ and $B_{t}^{2}$ are two independent Brownian motions ; and $\sigma_{i}$ stand for two standard deviations that are arbitrary fixed. Thereafter, we denote by $\mathbf{X}_{t}^{\mathbf{x}}$ the stochastic process $\mathbf{X}_{t}$ starting from the point $\mathbf{x}$ at $t=0$, i.e. $\mathbf{X}_{0}^{\mathbf{x}}=\mathbf{x}$.

The aim is to feature the stochastic scalar field $\Phi(\mathbf{x}, t)$ resulting from the action of the stochastic transport $\mathbf{X}_{t}$ onto a prescribe scalar field $\phi(\mathbf{x}, 0)$. Similarly to the deterministic transport where the time evolution of the field is defined through its Lagrangian description, the stochastic transport is defined for a particular sample $\omega$ by

$$
\Phi\left[\mathbf{X}_{t}^{\mathbf{x}}(\omega), t\right]=\phi(\mathbf{x}, 0)
$$

To provide a valid field at time $t$, this requires that the $\operatorname{map} \mathbf{X}_{t}(\omega): \mathbf{x} \longmapsto \mathbf{X}_{t}^{\mathbf{x}}(\omega)$ is one-to-one, which is not necessarily the case : it may happen that two distinct initial positions $x_{1}$ and $x_{2}$ leads to the same position at $t$ i.e.
$\mathbf{y}=\mathbf{X}_{t}^{\mathbf{x}_{1}}(\omega)=\mathbf{X}_{t}^{\mathbf{x}_{2}}(\omega)$ and then $\Phi(\mathbf{y}, t)$ should take two distinct values $\phi\left(\mathbf{x}_{1}, 0\right)$ and $\phi\left(\mathbf{x}_{2}, 0\right)$. To prevent from this failure, we have to assume that the map $\mathbf{X}_{t}(\omega)$ realises a diffeomorphism, defining by this way a stochastic flow (Kunita, 1990). Since $\mathbf{n}(\mathbf{x}, t)$ and $\mathbf{n}^{\perp}(\mathbf{x}, t)$, Eq.(9), are Lipshitzian fields near the zero level, then the process Eq.(9) defines a stochastic flow in a sufficiently large open set including the zero level. We have to rephrase the problem to obtain a more tractable formulation. This is done by introducing the pseudo-inverse flow $\tilde{\mathbf{X}}_{t}$ as follows. For an infinitesimal time, the antecedent point of $\mathbf{x}$ is $\mathbf{x}-d \mathbf{X}_{t}^{\mathbf{x}}$ so

$$
\begin{aligned}
\Phi(\mathbf{x}, \delta t) & =\phi\left(\mathbf{x}-d \mathbf{X}_{t}^{x}, 0\right) \\
& =\phi(\mathbf{x}, 0)-\nabla \phi \cdot d \mathbf{X}_{t}^{x}+\frac{1}{2} \phi^{\prime \prime}:\left(d \mathbf{X}_{t}^{\mathbf{x}}\right)^{T} \bullet d \mathbf{X}_{t}^{\mathbf{x}} \\
& =\phi(\mathbf{x}, 0)+\nabla \phi \cdot d \tilde{\mathbf{X}}_{t}^{\mathbf{x}}+\frac{1}{2} \phi^{\prime \prime}:\left(d \tilde{\mathbf{X}}_{t}^{\mathbf{x}}\right)^{T} \bullet d \tilde{\mathbf{X}}_{t}^{\mathbf{x}}, \\
& =\phi\left(\tilde{\mathbf{X}}_{\delta t}^{\mathbf{x}}, 0\right),
\end{aligned}
$$

where $\tilde{\mathbf{X}}_{t}^{x}$ is the process defined by

$$
d \tilde{\mathbf{X}}_{t}=-\mathbf{c} d t+\sigma_{1} \mathbf{n} d B_{t}^{1}+\sigma_{2} \mathbf{n}^{\perp} d B_{t}^{2}
$$

- denotes the matrix product operator with the additionnal algebraic rules $d t \bullet d t=d t \bullet d B_{t}^{i}=d B_{t}^{i} \bullet d t=0$ and $d B_{t}^{i} \bullet d B_{t}^{j}=\delta_{i j} d t$ using the Kroenecker symbol $\delta_{i j}$ (Oksendal, 2003), and the operator ":" denotes the matrix double contraction operator. Hence, it follows that

$$
\Phi(\mathbf{x}, t)=\phi\left(\tilde{\mathbf{X}}_{t}^{\mathbf{x}}, 0\right)
$$

which is dual of Eq.(10). Defining $\phi(\mathbf{x}, t)=\mathbb{E}[\Phi(\mathbf{x}, t)]=$ $\mathbb{E}\left[\phi\left(\tilde{\mathbf{X}}_{t}^{\mathbf{x}}, 0\right)\right]$ the average field, we are now able to use the backward Kolmogorov equation (Oksendal, 2003) so

$$
\partial_{t} \phi=\mathcal{L} \phi
$$


where $\mathcal{L}$ is the differential operator defined by

$$
(\mathcal{L} f)(\mathbf{x})=-(\mathbf{c} \cdot \mathbf{n})|\nabla f|+\frac{\sigma_{2}^{2}}{2} \kappa|\nabla f|+\frac{\sigma_{1}^{2}}{2} \frac{\nabla f^{T} f^{\prime \prime} \nabla f}{|\nabla f|^{2}} .
$$

Thus, when $\sigma_{1}$ is null, the dynamic of $\phi$ is given by the MHJE

$$
\partial_{t} \phi+\left(\mathbf{c} \cdot \mathbf{n}-\frac{\sigma_{2}^{2}}{2} \kappa\right)|\nabla \phi|=0
$$

that corresponds to the deterministic dynamic Eq.(8) of $\phi$, where $\varepsilon=\frac{1}{2} \sigma_{2}^{2}$. When $\sigma_{1}=\sigma_{2}=\sigma$, the dynamic is then given by

$$
\partial_{t} \phi+(\mathbf{c} \cdot \mathbf{n})|\nabla \phi|=\frac{\sigma^{2}}{2} \Delta \phi
$$

Thanks to this stochastic dynamics, an ensemble of $N$ contours $\mathcal{C}_{t}^{k}, k \in[1, N]$, can be constructed as the zero levels of an ensemble of level functions $\phi^{k}(\mathbf{x}, t)$ evolved by the dynamics Eq.(9). This is equivalent to phe $\frac{1}{N-1} \sum_{i=1: N}\left(\frac{1}{n-1} \sum_{x \in \mathcal{C}^{(i)}} w^{(i)}((d \mathbf{X}(x, k)-\mathbf{c}(x, k) d t) \cdot \mathbf{n})^{2}\right)$, deterministic time evolution of the expectation. Moreover, it results from Eq.(15) and Eq.(16) that the average field $\phi(\mathbf{x}, t)$ is solution of regularizing PDEs. This prevents from
singularity developments and secures the robustness of $\sigma_{\text {th }}^{2} \overline{\overline{\mathbf{e}}} \frac{1}{N-1} \sum_{i=1: N}\left(\frac{1}{n-1} \sum_{x \in \mathcal{C}^{(i)}} w^{(i)}\left((d \mathbf{X}(x, k)-\mathbf{c}(x, k) d t) \cdot \mathbf{n}^{\perp}\right)^{2}\right)$. ensemble method based on the stochastic transport Eq.(9).

Note that in practice, after a time step, the resulting potential functions do not remain signed distance functions. Thus, each potential function is regularly reinitialized to a signed distance function.

\section{Appendix B : tuning of the diffusion coefficients}

We propose to estimate the value of the noise diffusion coefficients. The dynamics defined through equation (8) involves two diffusion coefficients related to the uncertainty associated to the curve motion. These parameters $\sigma_{1}$ and $\sigma_{2}$ can be derived from the displacement field between two consecutive images, as follows. Let $d \mathbf{X}(x, k)$ be the displacement of point $x$ at time $k$ to its corresponding position at time $k+1$, according to the evolution of the implicit function $\phi$ conditioned on the past observations. We assume that this displacement field is a noisy version of $\mathbf{c}$,

$$
d \mathbf{X}(x, k)=\mathbf{c}(x, k) d t+\sigma_{1} \mathbf{n} d B_{k}^{1}+\sigma_{2} \mathbf{n}^{\perp} d B_{k}^{2},
$$

with noises along the normal and the tangent of $\phi$ levellines having same characteristics as those of the object. We are therefore making here the assumption that the noises associated with the level set displacement and the curve noises are collinear and have the same variances. Furthermore, we assume that the transport velocity field is such that $\mathbf{c}(x, t)=\mathbb{E}(d X(x, k)) / d t$. The empirical covariances with respect to the filtering law of this observed displacement along the curve normal and tangent provide an estimation of the noise variances $\sigma_{1}^{2}$ and $\sigma_{2}^{2}$ :

In practice, $d \mathbf{X}(x, t)$ is supplied by the optical flow measurements and the expectation are approached through empirical mean. For the time interval between the two first images, the values of these parameters are computed from the initial motion field used to initialize our filter.

\section{References}

Atger, F., 2001 : Verification of intense precipitation forecasts from single models and ensemble prediction systems. Nonlin. Proc. Geophys., 8, 401-417.

Avenel, C., Mémin E., Perez P., 2009 : Tracking closed curves with non-linear stochastic filters. Conf. on Scale Space and Variational Methods (SSVM'09), pp. 576-587, Voss, Norway.

Avenel, C., Mémin E., Perez P., 2014 : Stochastic Level Set Dynamics to Track Closed Curves Through Image Data. Journal of Mathematical Imaging and Vision, 49, Issue 2, 296-316.

Baehr, C. and Pannekoucke, O., 2011 : Some Issues and results on the EnKF and particule filters for meteorological models. 
chapter in Chaotic Systems : Theory and Applications- C. H. Skiadas and I. Dimotikalis (Editors) World Scientific (Publisher).

Baldwin, M.E. and J.S. Kain, 2006 : Sensitivity of several performance measures to displacement error, bias, and event frequency. Wea. Forecasting, 21, 636-648.

Ben Bouallègue, Z., S. E. Theis and C. Gebhardt, 2013 : Enhancing COSMO-DE ensemble forecasts by inexpensive techniques. Meteorol. Z., 22, 49-59.

Bhattacharyya, A. 1943 : On a measure of divergence between two statistical populations defined by their probability distributions. Bulletin of the Calcutta Mathematical Society, 35, 99-109.

Bouttier, F., Nuissier O., Vié B. and Raynaud L., 2012 : Impact of stochastic physics in a convection-permitting ensemble. Mon. Wea. Rev., 140, 3706-3721.

Bouttier, F., Raynaud L., Nuissier O. and Ménétrier B., 2015 : Sensitivities of the AROME ensemble to initial and surface perturbations during HyMeX. Quart. J. Roy. Meteor. Soc., DOI : 10.1002/qj.2622.

Bowler, N. E. H., C. E. Pierce, and A. Seed, 2006 : STEPS : A probabilistic precipitation forecasting scheme which merges an extrapolation nowcast with downscaled NWP. Quart. J. Roy. Meteor. Soc., 132, 2127-2155.

Briggs, W.M. and R.A. Levine, 1997 : Wavelets and field forecast verification. Mon. Wea. Rev., 125, 1329-1341.

Chan, T. and Vese, L., 2001 : Active contours without edges. IEEE Transactions on Image Processing, 10, 266-277.

Damrath, U., 2004 : Verification against precipitation observations of a high density network what did we learn? Intl. Verification Methods Workshop, 15-17 September 2004, Montreal, Canada.

Dance, S., E. Ebert and D. Scurrah, 2010 : Thunderstorm strike probability nowcasting, J. Atmos. Oceanic Technol., 27, 79-93.

Davis, C., B. Brown, and R. Bullock, 2006 : Object-based verification of precipitation forecasts. Part I : Methods and application to mesoscale rain areas. Mon. Wea. Rev., 134, 17721784.

Del Moral P., 2004 : Feynman-Kac Formulae. Genealogical and Interacting Particle Systems with Applications, Probability and its Applications, Springer-Verlag, New York, 2004.

Doucet, A., S.J. Godsill and C. Andrieu, 2000 : On sequential Monte-Carlo sampling methods for Baysian filtering. Statist. Comput., 10, 197-208.
Ebert, E.E. 2008 : Fuzzy verification of high resolution gridded forecasts : A review and proposed framework. Meteorol. Appl., 15, $51-64$.

Ebert, E.E. and J.L. McBride, 2000 : Verification of precipitation in weather systems : Determination of systematic errors. J. Hydrology, 239, 179-202.

Gordon, N.D., D.J. Salmond, and A.F.M. Smith, 1993 : Novel approach to nonlinear/non-Gaussian Bayesian state estimation. IEEE Proc F, 140, 107-113.

Keil, C. and G.C. Craig, 2007 : A displacement-based error measure applied in a regional ensemble forecasting system. Mon. Wea. Rev., 135, 3248-3259.

Keil, C. and G.C. Craig, 2009 : A displacement and amplitude score employing an optical flow technique. Wea. Forecasting, 24, $1297-1308$.

Marzban, C. and S. Sandgathe, 2006 : Cluster analysis for verification of precipitation fields, Wea. Forecasting, 21, 824-838.

Marzban, C. and S. Sandgathe, 2008 : Cluster analysis for object-oriented verification of fields : A variation. Mon. Wea. Rev., $136,1013-1025$.

Marzban C., S. Sandgathe., 2010 : Optical Flow for Verification. Weather and Forecasting, 25 (5), 1479-1494.

Michaes, A.C., N.I. Fox, S.A. Lack and C.K. Wikle, 2007 : Cell identification and verification of QPF ensembles using shape analysis techniques. J. Hydrol., 343, 105-116.

Nachamkin, J.E., 2004 : Mesoscale verification using meteorological composites. Mon. Wea. Rev., 132, 941-955.

Oksendal, B., 2003 : Stochastic differential equations, An Introduction with Applications. Springer.

Osher, S., and Sethian, J., 1988 : Fronts Propagating with Curvature-Dependent Speed : Algorithms Based on HamiltonJacobi Formulations. Journal of Computationnal Physics, 79, 1249.

Pannekoucke, O. and Vezard, L., 2010 : Stochastic integration for the heterogeneous correlation modeling using a diffusion equation. Mon. Wea. Rev., 138, 3356-3365.

Papadakis, N., Mémin, E., 2008 : A variational technique for time consistent tracking of curves and motion. Journal of Mathematical Imaging and Vision, 31 (1), 81-103.

Raynaud L., and Bouttier F., 2015 : Comparison of initial perturbation methods for ensemble prediction at convective scale. Quart. J. Roy. Meteor. Soc., DOI : 10.1002/qj.2686. 
Raynaud L., O. Pannekoucke, P. Arbogast and Bouttier F., 2015 : Application of a Bayesian weighting for short-range lagged ensemble forecasting at the convective scale. Quart. J. Roy. Meteor. Soc., 141, 459-468.

Roberts, N.M. and H.W. Lean, 2008 : Scales-selective verification of rainfall accumulations from high-resolution forecasts of convective events. Mon. Wea. Rev., 136, 78-97.

Sethian, J., 1996 : Level set methods : an act of violence evolving interfaces in geometry, fluid mechanics, computer vision and materials sciences.

Seity Y., P. Brousseau, S. Malardel, G. Hello, P. Bénard, F. Bouttier, C. Lac, V. Masson, 2011; The AROME-France Convective-Scale Operational Model, Monthly Weather Review, 139, 976-991.

Steinbrücker F., T. Pock and D. Cremers, 2009 : Large displacement optical flow computation without warping. In Proc. International Conference on Computer Vision.

Van Leeuwen, P.J. , 2009 : Particle Filtering in Geophysical Systems, Mon. Wea. Rev., 137, 4089-4114.

Wernli H., M. Paulat, M. Hagen and C. Frei, 2008 : SAL a novel quality measure for the verification of quantitative precipitation forecasts. Mon. Wea. Rev., 136, 4470-4487.

Yates, E., S. Anquetin, V. Ducrocq, J.-D. Creutin, D. Ricard and K. Chancibault, 2006 : Point and areal validation of forecast precipitation fields. Meteorol. Appl., 13, 11-20.

Zepeda-Arce, J., E. Foufoula-Georgiou, and K.K. Droegemeier, 2000 : Space-time rainfall organization and its role in validating quantitative precipitation forecasts. J. Geophys. Res., 105 (D8), 10129-10146. 